\title{
Estudo retrospectivo de diagnósticos post-mortem de cães e gatos necropsiados no Setor de Patologia Animal da Universidade Federal do Piauí, Brasil de 2009 a 2014
}

\author{
Retrospective study of post-mortem diagnosis of dogs and cats necropsied in the Animal Pathology \\ Sector of Piauí Federal University, Brazil from 2009 to 2014
}

\author{
Emanuelle Karine Frota BATISTA ${ }^{1}$; Lidiany Viana PIRES ${ }^{1}$; Dayane Francisca Higino MIRANDA ${ }^{1}$; Werner \\ Rocha ALBUQUERQUE²; Alinne Rosa de Melo CARVALHO²; Lucilene dos Santos SILVA³ Silvana Maria \\ Medeiros de Souza SILVA ${ }^{1}$
}

\author{
${ }^{1}$ Universidade Federal do Piauí, Centro de Ciências Agrárias, Programa de Pós-graduação em Ciência Animal, Teresina - PI, Brasil \\ ${ }^{2}$ Universidade Federal do Piauí, Centro de Ciências Agrárias, Patologia Animal, Teresina - PI, Brasil \\ ${ }^{3}$ Médica veterinária, Teresina - PI, Brasil
}

\begin{abstract}
Resumo
O conhecimento das principais causas de óbito em cães e gatos fornece subsídios para o monitoramento, planejamento e avaliação de medidas que visam reduzir o percentual de óbito desses animais em uma dada localidade. $\mathrm{O}$ presente trabalho compilou os diagnósticos post-mortem de cães e gatos necropsiados no Laboratório de Patologia Animal - da Universidade Federal do Piauí (UFPI), Estado do Piauí, Brasil, no período de agosto de 2009 a agosto de 2014, estabelecendo a frequência das doenças que culminaram com o óbito dos animais. Nesse período foram necropsiados 361 cães e 86 gatos. Dos cães, 56,7\% eram machos e $43,3 \%$ fêmeas. Em relação à idade no momento do óbito, 29,4\% tinham menos de um ano; $27,7 \%$ entre 1,1 a 5 anos; 23,3\% de 5,1 a 10 anos e 9,1\% acima de 10,1 anos. Em relação aos felinos, 61,6\% eram machos e 38,4\% eram fêmeas, dos quais $29,1 \%$ tinham menos de um ano; $39,5 \%$ de 1,1 a 5 anos; $18,6 \%$ de 5,1 a 10 anos e 2,3\% acima de 10,1 anos. Nos cães as principais causas de óbito foram distúrbios infecciosos (23,8\%), doenças degenerativas (14,4\%), distúrbios circulatórios $(10,2 \%)$ e neoplasias $8,6 \%$. Em gatos, os distúrbios infecciosos $(18,6 \%)$, urinários $(15,1 \%)$, traumáticos $(8,1 \%)$ e neoplasias $(8,1 \%)$ foram as principais causas de morte. Conclui-se que a principal causa de morte, tanto em cães quanto gatos, diagnosticada no setor de Patologia Animal - UFPI foram as doenças infecciosas, estes resultados contribuem para que o clínico dedique maior atenção a essas enfermidades, visando adoção de medidas profiláticas que reduzirão a sua ocorrência nos animais de companhia da região estudada.
\end{abstract}

Palavras-chave: Causa mortis. Epidemiologia. Necropsia. Óbito.

\begin{abstract}
Knowledge of the main causes of death in dogs and cats provides grants for monitoring, planning and evaluation of measures to reduce the percentage of death of these animals. Therefore, the present paper was developed to analyze the diagnostic postmortem of dogs and cats in Animal Pathology Laboratory - UFPI from August 2009 to August 2014, establishing the frequency of causes that led to the animal deaths. $361 \mathrm{dogs}$ and 86 cats were necropsied in this period. Of the dogs, $56.7 \%$ were males and $43.3 \%$ females. Regarding age at death, in the group of dogs, $29.4 \%$ had less than 1 year; $27.7 \%$ between 1.1 to 5 years; $23.3 \% 5.1$ to 10 years and $9.1 \%$ over 10.1 years. In the group of cats, $61.6 \%$ were male and $38.4 \%$ female, of which $29.1 \%$ were less than 1 year; $39.5 \% 1.1$ to 5 years; $18.6 \% 5.1$ to 10 years and $2.3 \%$ over 10.1 years. The main causes of death in dogs were infectious disorders (23.8\%), degenerative diseases (14.4\%), circulatory disorders (10.2\%) and neoplasms 8.6\%. In cats, infectious disorders $(18.6 \%)$, urinary $(15.1 \%)$, trauma $(8.1 \%)$ and neoplasms $(8.1 \%)$ were the leading causes of death. It is concluded that the main causes of death in both pet animal species, diagnosed in animal-UFPI Pathology sector, were infectious diseases. Local veterinary services should be made aware of these results, leading to measures for paying more attention to these diseases and the adoption of prophylactic measures to reduce the occurrence of such diseases in pets.
\end{abstract}

Keywords: Cause of mortis. Epidemiology. Autopsy. Death. 
Correspondência para:

Emanuelle Karine Frota Batista

Centro de Ciências Agrárias/UFPI

Av. Universitária, $\mathrm{s} / \mathrm{n}^{\circ}$

CEP 64049-550, Teresina, PI, Brasil

e-mail: emanuellefrota@yahoo.com.br

Recebido: 29/01/2015

Aprovado: 09/02/2016

\section{Introdução}

A necropsia é o exame externo e interno de um cadáver que tem a finalidade de estabelecer a causa da morte do animal. Além de satisfazer a curiosidade do proprietário, criador ou veterinário responsável, a necropsia fornece informações sobre as questões clínicas que não foram definidas quando o paciente ainda estava vivo e direciona o diagnóstico $\mathrm{e}$ tratamento de casos futuros (HULL et al., 2007; GONÇALVES; SALGADO, 2011).

$\mathrm{O}$ esclarecimento das principais causas de morte de cães e gatos em uma determinada localidade pode fornecer elementos que propiciem o monitoramento, planejamento e a avaliação de medidas de controle destinadas a reduzir o percentual de óbitos $\mathrm{e}$ contribuir para a melhoria da qualidade de vida dos animais (BONNETT et al., 2005). O conhecimento da frequência de ocorrência das diferentes doenças que afetam os animais serve como um guia para o estabelecimento da lista de diagnósticos diferenciais frente à determinada manifestação clínica, laboratorial ou anatomopatológica (FIGHERA et al., 2008). Uma das principais funções do médico veterinário é a de atuar de forma preventiva, conscientizando a população sobre o bem-estar dos animais e temas relacionados à saúde pública (LOSS et al., 2012). Os profissionais médicos veterinários que atuam em uma determinada localidade devemos conhecer as principais causas de morte de animais de companhia na região e estar conscientes das diferenças existentes entre as raças, sexo, porte ou idade, para aconselharem os futuros proprietários sobre medidas de saúde que possam minimizar ou pelo menos retardar o aparecimento de determinadas doenças (PROSCHOWSKY et al., 2003).

Há poucos estudos disponíveis sobre os registros dos serviços de diagnóstico anátomo patológico em populações de cães e gatos, contudo as doenças de origem infecciosa, traumática, degenerativa ou neoplásica, têm sido descritas como as principais causas de morte espontânea ou como justificativa para a realização da eutanásia (FIGHERA et al, 2008; TRAPP et al., 2010; FLEMING et al., 2011). Os processos neoplásicos têm apresentado uma maior incidência na população geriátrica (MARTINS et al., 2011).

No Estado do Piauí ainda não foram efetuadas análises das causas da mortalidade de cães e gatos submetidos a necropsias. Portanto, o presente trabalho foi delineado para compilar os registros de necropsias de cães e gatos efetuadas no Laboratório de Patologia Animal, da Universidade Federal do Piauí, durante o período de agosto de 2009 à agosto de 2014, de modo a permitir o estabelecimento da relação das doenças ou distúrbios que culminaram com o óbito dos animais.

\section{Material e Métodos}

Foram analisadas todas as requisições de necropsia e os respectivos relatórios anatomopatológicos de cães e gatos, realizadas no Laboratório de Patologia Animal, do Departamento de Clínica e Cirurgia Veterinária, da Universidade Federal do Piauí, no período de agosto de 2009 a agosto de 2014. Os animais atendidos neste setor são provenientes da cidade de Teresina, capital do estado do Piauí. As requisições de necropsia continham o histórico clinico do animal, informando os resultados dos exames complementares (hemograma, bioquímico, sorológico, parasitológico), o diagnóstico clínico e o tratamento realizado. Os registros foram organizados em tabelas, que permitiram a associação das diferentes patologias com as variáveis sexo, raça, idade e diagnóstico da causa de morte. Foi considerado um único diagnóstico definitivo para 
cada caso (BONNETT et al., 2005; BENTUBO et al., 2007; FIGHERA et al., 2008). Quando não havia evidências que permitissem o estabelecimento de um diagnóstico definitivo, os casos foram considerados como inconclusivos. Tais situações estavam associadas à ausência completa de lesões morfológicas ou à presença apenas de lesões incidentais.

Os diagnósticos da causa de morte foram classificados em doenças infecciosas (quadro sugestivo); doenças parasitárias, distúrbios causados por agentes físicos (traumas), neoplasias, doenças degenerativas, distúrbios circulatórios, doenças metabólicas e endocrinológicas, doenças nutricionais e intoxicações (TRAPP et al., 2010). As doenças que não puderam ser classificadas em nenhum desses tópicos foram agrupadas sob a expressão "outros distúrbios", (MOORE et al., 2001; PROSCHOWSKY et al., 2003). Os animais foram subdivididos em cinco grupos etários: filhotes (até um ano de idade), jovens (a partir de um ano até cinco anos), adultos (a partir de cinco anos e até dez anos), idosos (acima de dez anos) e os de idade não identificada (MENEZES et al., 2011).

Concluída a classificação foram calculadas as frequências de ocorrência em cada grupo e para cada condição em relação ao total de animais necropsiados. A avaliação estatística dos resultados foi realizada com base na análise da existência de associação entre as variáveis: diagnósticos necroscópicos, raça e faixa etária com o emprego do teste do Qui-quadrado software GraphPad Prism 6. O nível de confiança adotado foi de 0.05

\section{Resultados}

Nos arquivos do Laboratório de Patologia Animal da Universidade Federal do Piauí (UFPI) no período compreendido entre agosto de 2009 e agosto de 2014, foram encontrados 361 protocolos de necropsia de cães e 86 de gatos.

Do total de cães, $56,7 \%$ correspondiam a machos e $43,3 \%$ a fêmeas. Desta espécie, $29,4 \%$ foram incluídos na faixa etária até 1 ano; $27,7 \%$ de 1,1 a 5 anos; $23,3 \%$ de 5,1 a 10 anos; 9,1\% acima de 10,1 anos e 12,5\% com idade indeterminada. Em relação ao total de gatos, $61,7 \%$ eram machos e 38,4\% eram fêmeas. Com relação à idade destes animais, $33,7 \%$ tinham menos de 1 ano; $39,5 \%$ de 1,1 a 5 anos; $11,6 \%$ de 5,1 a 10 anos; $1,2 \%$ acima de 10,1 anos e $13,9 \%$ com idade indeterminada.

Dos 361 cães necropsiados Laboratório de Patologia Animal da Universidade Federal do Piauí (UFPI) no período compreendido entre agosto de 2009 e agosto de 2014, foi estabelecida a causa da morte em 340/361 (94,2\%), casos denominados como conclusivos. A distribuição dos casos em ordem decrescente de frequência de ocorrência foi: $1^{\circ}$ ) doenças infecciosas; $2^{\circ}$ ) doenças degenerativas; $3^{\circ}$ ) distúrbios circulatórios; $4^{\circ}$ ) neoplasias; $5^{\circ}$ ) doenças nutricionais; $6^{\circ}$ ) intoxicações; $7^{\circ}$ ) distúrbios causados por agentes físicos; $8^{\circ}$ ) doenças parasitárias; $9^{\circ}$ ) doenças metabólicas e endocrinológicas; e $10^{\circ}$ ) "outros distúrbios" (causas multifatoriais e/ou idiopáticas) (Tabela 1).

A frequência de ocorrência de óbitos devido a doenças infecciosas em cães foi de 86/361 (23,82\%). Destas parvovirose e a erliquiose foram as afecções mais frequentes, com, respectivamente 44/86 $(51,16 \%)$ e $35 / 86(40,69 \%)$ casos. A análise da variável faixa etária do cão no momento do óbito revelou que $37 / 86(43,02 \%)$ apresentavam idade inferior a um ano, ocorrendo nessa faixa uma associação positiva com óbitos devido às doenças infecciosas $\left(\chi^{2}=15,25 ; \mathrm{P}=\right.$ 0,0042).

Em relação às doenças degenerativas, a frequência de ocorrência de óbitos foi de 52/361 (14,4\%), sendo que $14 / 52(26,92 \%)$ dos cães apresentavam idade entre 1,1 e 5 anos, havendo associação positiva entre essa faixa etária e a morte por doenças degenerativas $\left(\chi^{2}=18,79 ; \mathrm{P}=0,009\right)$. A principal doença circulatória que acometeu os cães foi o choque hipovolêmico, 28/37 (75,67\%). As neoplasias foram responsáveis por 31/361 (8,58\%) dos óbitos em cães, sendo em 11/31 $(35,48 \%)$ dos casos já havia o desenvolvimento de metástases, não podendo ser definido o ponto de 
Tabela 1 - Diagnósticos Post-mortem de cães necropsiados no Laboratório de Patologia Animal da Universidade Federal do Piaui, Brasil, no período de agosto de 2009 a agosto de 2014 segundo gênero, raça e idade

\begin{tabular}{|c|c|c|c|c|c|c|c|c|c|c|c|c|c|c|c|c|c|c|c|}
\hline \multirow{3}{*}{ CAUSA DA MORTE } & \multicolumn{4}{|c|}{ Gênero } & \multicolumn{4}{|c|}{ Raça } & \multicolumn{10}{|c|}{ Idade } & \multirow{3}{*}{ TOTAL } \\
\hline & \multicolumn{2}{|c|}{$\mathrm{M}$} & \multicolumn{2}{|c|}{$\mathrm{F}$} & \multicolumn{2}{|c|}{ Pura } & \multicolumn{2}{|c|}{ SRD } & \multicolumn{2}{|c|}{$\leq 1$} & \multicolumn{2}{|c|}{$1,1-5$} & \multicolumn{2}{|c|}{$5-10$} & \multicolumn{2}{|c|}{$>10$} & \multicolumn{2}{|c|}{$\mathrm{ND}^{*}$} & \\
\hline & No & $\%$ & No & $\%$ & $\mathrm{No}^{\circ}$ & $\%$ & $\mathrm{~N}^{\circ}$ & $\%$ & No & $\%$ & $\mathrm{No}^{\circ}$ & $\%$ & $\mathrm{~N}^{o}$ & $\%$ & $\mathrm{~N}^{\circ}$ & $\%$ & $\mathrm{~N}^{\circ}$ & $\%$ & \\
\hline Doenças infecciosas & 56 & 65,1 & 30 & 34,9 & 43 & 50 & 43 & 50 & 37 & 43,02 & 25 & 29,1 & 15 & 17,4 & 2 & 2,3 & 7 & 8,1 & 86 \\
\hline Doença degenerativa & 26 & 50,0 & 26 & 50,0 & 35 & 67,3 & 17 & 32,7 & 9 & 17,3 & 14 & 26,92 & 11 & 21,2 & 4 & 7,7 & 14 & 26,9 & 52 \\
\hline Distúrbios circulatórios & 15 & 40,5 & 22 & 59,5 & 19 & 51,4 & 18 & 48,6 & 7 & 18,9 & 16 & 43,2 & 11 & 29,7 & 2 & 5,4 & 1 & 2,7 & 37 \\
\hline Neoplasias & 15 & 48,4 & 16 & 51,6 & 17 & 54,8 & 14 & 45,2 & 1 & 3,2 & 5 & 16,1 & 12 & 38,7 & 12 & 38,7 & 1 & 3,2 & 31 \\
\hline Doença nutricional & 13 & 68,4 & 6 & 31,6 & 17 & 89,5 & 2 & 10,5 & 15 & 78,9 & 1 & 5,3 & 0 & 0,0 & 1 & 5,3 & 2 & 10,5 & 19 \\
\hline Intoxicações & 11 & 61,1 & 7 & 38,9 & 10 & 55,6 & 8 & 44,4 & 2 & 11,1 & 8 & 44,4 & 4 & 22,2 & 0 & 0,0 & 4 & 22,2 & 18 \\
\hline Distúrbios causados por agentes físicos & 13 & 76,5 & 4 & 23,5 & 9 & 52,9 & 8 & 47,1 & 8 & 47,1 & 6 & 35,3 & 1 & 5,9 & 1 & 5,9 & 1 & 5,9 & 17 \\
\hline Doenças parasitarias & 10 & 62,5 & 6 & 37,5 & 7 & 43,8 & 9 & 56,3 & 3 & 18,8 & 3 & 18,8 & 6 & 37,5 & 2 & 12,5 & 2 & 12,5 & 16 \\
\hline Doenças metabólicas endocrinológicas & 0 & 0,0 & 7 & 100,0 & 5 & 71,4 & 2 & 28,6 & 0 & 0 & 0 & 0,0 & 4 & 57,1 & 1 & 14,3 & 2 & 28,6 & 7 \\
\hline "Outros distúrbios" & 33 & 58,9 & 23 & 41,1 & 32 & 57,1 & 24 & 42,9 & 16 & 28,6 & 18 & 32,1 & 15 & 26,8 & 7 & 12,5 & & 0,0 & 56 \\
\hline Inconclusivos & 13 & 65,0 & 9 & 45,0 & 12 & 54,5 & 10 & 45,5 & 8 & 36,4 & 4 & 18,2 & 5 & 22,7 & 1 & 4,5 & 4 & 18,2 & 22 \\
\hline Total & 205 & & 156 & & 206 & & 155 & & 106 & & 100 & & 84 & & 33 & & 38 & & 361 \\
\hline
\end{tabular}

${ }^{*} \mathrm{ND}$ : não determinada 
origem. O linfoma e o mesotelioma foram as principais neoplasias que culminaram com a morte dos cães, correspondendo, respectivamente a 5/31 $(16,12 \%)$ e $4 / 31(12,9 \%)$ dos casos. A idade da maioria dos cães $25 / 31(80,64 \%)$ no momento do óbito foi superior a cinco anos $\left(\chi^{2}=47,16 ; \mathrm{P}<0,0001\right)$.

$\mathrm{Na}$ categoria de doenças nutricionais, 19/361 $(5,26 \%)$ dos cães apresentavam um quadro de anemia e hipoproteinemia, afetando 15/19 (78,94\%) dos cães com menos de um ano de idade. O restante dos 361 casos foi categorizado em intoxicações, afetando 18/361 (4,98\%) dos animais; doenças causadas por agentes físicos acometeram 17/361 (4,7\%) dos cães, sendo 7/17 (41,17\%) destes devido a traumas decorrentes de brigas; doenças parasitárias afetaram 16/361 (4,43\%) dos cães, sendo que 8/16 (50\%) desses animais apresentava leishmaniose; as doenças metabólicas e endocrinológicas acometeram 7/361 $(1,93 \%)$ dos animais, com 5/7 (71,42\%) destes apresentando piometra. Não houve associação positiva $(P>0,05)$ entre os demais diagnósticos e a faixa etária dos animais no momento do óbito. Em relação às raças dos animais, só foi constatada associação positiva com óbitos decorrentes de doença nutricional $\left(\chi^{2}=8,598 ; \mathrm{P}=0,0034\right)$

Dos 86 gatos necropsiaos, a causa da morte foi firmada em 80 (93\%) (Tabela 2).

Dos 86 gatos necropsiados o diagnóstico presuntivo de doenças infecciosas incluiu 16/86 $(18,6 \%)$ dos animais, sendo a peritonite infecciosa felina a afecção mais frequente, ocorrendo em 11/16 $(68,7 \%)$ destes animais. Considerando a faixa etária dos animais no momento do óbito, $8 / 11$ (72,7\%) dos felinos com peritonite apresentaram idade entre um e cinco anos.

As doenças do sistema urinário foram responsáveis por 13/86 $(15,1 \%)$ dos óbitos em felinos. Dentre estas, a doença do trato urinário inferior foi a afecção mais prevalente, afetando 7/13 (53,8\%) dos animais. A neoplasia foi observada em 7/86 (8,14\%), com desenvolvimento de metástases em 5/7 (71,42\%) dos casos, afetando 5/7 (71,42\%) dos animais entre cinco e dez anos de idade, havendo associação positiva entre essa faixa etária e óbitos decorrentes de neoplasias $\left(\chi^{2}=27,39 ; \mathrm{P}<0,0001\right)$. Dentre as doenças causadas por agentes físicos, $71,5 \%$ dos felinos foram acometidos por traumas de diferentes origens.

As doenças degenerativas ocorreram em 6/86 (6,97\%) dos felinos. A doença nutricional afetou 6/86 $(6,97 \%)$ dos gatos, e 5/6 (83,3\%) destes morreram devido a um quadro de anemia e hipoproteinemia. A morte por intoxicação ocorreu em 6/86 (6,97\%) dos animais. Os demais casos foram categorizados como doenças circulatórias, que afetaram 4/86 (4,65\%) dos gatos; doenças parasitárias, que ocorreram em 2/86 $(2,3 \%)$ desses animais e doenças metabólicas, com $1 / 86(1,16 \%)$.

Não houve associação positiva $(\mathrm{P}>0,05)$ entre os demais diagnósticos e a faixa etária dos animais no momento do óbito, nem entre a raça dos animais diagnosticados.

\section{Discussão}

O número de cães submetidos a exame necroscópico nesse laboratório foi maior que o de gatos, isto deve-se ao fato de os cães serem mais populares como animais de companhia. Segundo dados atualizados da Associação Brasileira da Indústria de Produtos para Animais de Estimação ${ }^{1}$, há 37,1 milhões de cães e 21,3 milhões de gatos nos lares brasileiros.

O predomínio do óbito de cães decorrentes de doenças infecciosas assemelha-se ao observado em outros estudos conduzidos no Brasil, no estado de São Paulo (BENTUBO et al., 2007), Paraná (TRAPP et al., 2010) e Rio Grande do Sul (FIGHERA et al., 2008), que destacaram as doenças infecciosas ou parasitárias como as principais causas de óbitos em cães. Essa constatação pode estar relacionada às condições de higiene, a baixa adesão aos programas de vacinação e vermifugação, bem como, a negligência dos proprietários nacionais (BENTUBO et al., 2007;

\footnotetext{
${ }^{1}$ http://www.2pro.com.br/site/abinpet-preve-crescimento-defaturamento-de-82-para-setor-pet-que-deve-faturar-r-1647-bi-em-2014/
} 
Tabela 2 - Diagnósticos Post-mortem de animais da espécie felina necropsiados no Laboratório de Patologia Animal da Universidade Federal do Piauí, Brasil no período de agosto de 2009 a agosto de 2014, segundo gênero, raça e idade

\begin{tabular}{|c|c|c|c|c|c|c|c|c|c|c|c|c|c|c|c|c|c|c|c|}
\hline \multirow{3}{*}{ CAUSA DA MORTE } & \multicolumn{4}{|c|}{ Gênero } & \multicolumn{4}{|c|}{ Raça } & \multicolumn{10}{|c|}{ Idade } & \multirow{3}{*}{ TOTAL } \\
\hline & \multicolumn{2}{|c|}{$M$} & \multicolumn{2}{|c|}{$\mathrm{F}$} & \multicolumn{2}{|c|}{ Pura } & \multicolumn{2}{|c|}{ SRD } & \multicolumn{2}{|c|}{$\leq 1$} & \multicolumn{2}{|c|}{$1,1-5$} & \multicolumn{2}{|c|}{$5-10$} & \multicolumn{2}{|c|}{$>10$} & \multicolumn{2}{|c|}{$\mathrm{ND}^{*}$} & \\
\hline & $\mathrm{N}^{\mathrm{o}}$ & $\%$ & $\mathrm{~N}^{\circ}$ & $\%$ & $\mathrm{~N}^{\mathrm{o}}$ & $\%$ & $\mathrm{~N}^{o}$ & $\%$ & No & $\%$ & $\mathrm{~N}^{\mathrm{o}}$ & $\%$ & $\mathrm{~N}^{o}$ & $\%$ & $\mathrm{~N}^{\circ}$ & $\%$ & $\mathrm{~N}^{\mathrm{o}}$ & $\%$ & \\
\hline Doenças infecciosas & 9 & 56,25 & 7 & 43,25 & 4 & 25,0 & 12 & 75,0 & 3 & 18,75 & 10 & 62,5 & 0 & 0 & 0 & 0 & 3 & 18,75 & 16 \\
\hline Distúrbio urinário & 10 & 76,9 & 3 & 23,1 & 4 & 30,8 & 9 & 69,2 & 2 & 15,4 & 6 & 46,2 & 3 & 23,1 & 0 & 0 & 2 & 15,4 & 13 \\
\hline Neoplasias & 3 & 42,8 & 4 & 57,14 & 1 & 14,3 & 6 & 85,7 & 1 & 14,3 & 1 & 14,3 & 5 & 71,4 & 0 & 0 & 0 & 0 & 7 \\
\hline Distúrbios causados por agentes físicos & 6 & 85,7 & 1 & 14,29 & 1 & 14,3 & 6 & 85,7 & 4 & 57,1 & 3 & 42,9 & 0 & 0,0 & 0 & 0 & 0 & 0 & 7 \\
\hline Doença degenerativa & 4 & 66,6 & 2 & 33,33 & 2 & 33,3 & 4 & 66,7 & 2 & 33,3 & 4 & 66,7 & 0 & 0,0 & 0 & 0 & 0 & 0 & 6 \\
\hline Doença nutricional & 3 & 50 & 3 & 50 & 1 & 16,7 & 5 & 83,3 & 3 & 50,0 & 2 & 33,3 & 1 & 16,7 & 0 & 0 & 0 & 0 & 6 \\
\hline Intoxicações & 3 & 50 & 3 & 50 & 0 & 0,0 & 6 & 100,0 & 5 & 83,3 & 0 & 0,0 & 0 & 0,0 & 0 & 0 & 1 & 16,7 & 6 \\
\hline Distúrbios circulatórios & 3 & 75 & 1 & 25 & 0 & 0,0 & 4 & 100,0 & 2 & 50,0 & 0 & 0,0 & 1 & 25,0 & 0 & 0 & 1 & 25 & 4 \\
\hline Doenças parasitarias & 2 & 100 & 0 & 0 & 0 & 0,0 & 2 & 100,0 & 1 & 50,0 & 1 & 50,0 & 0 & 0,0 & 0 & 0 & 0 & 0 & 2 \\
\hline Doenças metabólicas e endocrinológicas & 0 & 0 & 1 & 100 & 0 & 0,0 & 1 & 100,0 & 0 & 0,0 & 1 & 100,0 & 0 & 0,0 & 0 & 0 & 0 & 0 & 1 \\
\hline "Outros distúrbios" & 7 & 61,5 & 5 & 38,5 & 5 & 38,5 & 7 & 61,5 & 0 & 0,0 & 4 & 30,8 & 6 & 46,1 & 2 & 15,4 & 0 & 7,7 & 12 \\
\hline Inconclusivos & 3 & 50 & 3 & 50 & 3 & 50,0 & 3 & 50,0 & 2 & 33,3 & 2 & 33,3 & 0 & 0 & 0 & 0 & 2 & 33,3 & 6 \\
\hline Total & 53 & & 33 & & 21 & & 65 & & 25 & & 34 & & 16 & & 2 & & 9 & & 86 \\
\hline
\end{tabular}


FIGHERA et al., 2008; TRAPP et al., 2010). Em outros países, como Dinamarca, Estados Unidos e Suécia, a ocorrência de óbitos de cães em consequência de doenças infecciosas ou parasitárias é baixa, pois a maioria dos cães é vacinada e revacinada anualmente, diferindo do que ocorre no Brasil, onde apenas uma pequena parcela de proprietários adere aos esquemas de vacinação e vermifugação completos para seus cães (CRAIG, 2001; MOORE et al., 2001; PROSCHOWSKY et al., 2003; BONNETT et al., 2005; GREENE; SCHULTZ, 2006). Medidas profiláticas como imunoprofilaxia são fundamentais para o controle de doenças infecciosas, entretanto, a falta de conhecimento, os aspectos sócio-econômicos e a negligência dos proprietários dificultam a aplicação de tais procedimentos (BENTUBO et al., 2007).

A prevalência de óbitos em cães decorrentes de doenças degenerativas foi superior aos resultados obtidos por Fighera et al. (2008) no Rio Grande do Sul, e Trapp et al. (2010) no Paraná, que relataram a ocorrência dessa causa de morte, respectivamente em, $7,1 \%$ e $8,63 \%$.

A frequência do óbito em cães por neoplasias observada nesse estudo aproxima-se do observado em outros relatos nacionais cujos valores oscilam entre $7,8 \%$ e $13,3 \%$ (BENTUBO et al., 2007; FIGHERA et al., 2008). No entanto, em muitos países como Estados Unidos, Dinamarca e Suécia as neoplasias são consideradas a principal causa de morte canina cuja frequência de ocorrência situa-se é de 14 a 39,5\% (CRAIG, 2001; MOORE et al., 2001; PROSCHOWSKY et al., 2003; BONNETT et al., 2005). Possivelmente, a diferença observada entre os países seja decorrente da menor expectativa de vida dos cães no Brasil, (BENTUBO et al., 2007).

As doenças infeciosas também se situaram entre as principais causas de morte em gatos, afetando principalmente animais jovens, havendo um segundo pico de incidência em idades mais avançadas (EGENVALL et al., 2009). A peritonite infeciosa felina (PIF) é uma das principais causas de morte em gatos em todas as idades (YOUNG, 2011).
Vários estudos têm apontado as doenças do trato urinário inferior como a causa de maior morbidade e mortalidade em felinos, afetando principalmente os animais idosos (FRANCEY; SCHWEIGHAUSER, 2008; GRAUER, 2009; SCHERK, 2012). Withrow (2007) na Inglaterra e North e Banks (2009) nos Estados Unidos consideram que as doenças do trato urinário inferior sejam a segunda causa de morte mais comum em felinos, e que as neoplasias ocupam a primeira posição nestes animais. As neoplasias descritas como mais comuns em felinos são as hematopoiéticas, seguidas de neoplasia de pele e tecidos subcutâneos e por último, neoplasia da glândula mamária (BLACKWOOD, 2013).

\section{Conclusões}

As principais causas de morte diagnosticadas em cães, no Setor de Patologia Animal da UFPI, foram em ordem decrescente: as doenças infecciosas, degenerativas, circulatórias e neoplasias. Nos gatos, as principais causas de morte, em ordem decrescente, foram: doenças infecciosas, doenças do trato urinário, neoplasias e doenças causadas por agentes físicos. Os resultados obtidos oferecem subsídios para adoção de medidas profiláticas aplicadas as populações de cães e gatos de companhia na região estudada. 


\section{Referências}

BENTUBO, H. D. L.; TOMAZ, M. A.; BONDAN, E. F.; LALLO, M. A. Expectativa de vida e causas de morte em cães na área metropolitana de São Paulo (Brasil). Ciência Rural, v. 37, n. 4, p. 1021-1026, 2007. doi: http://dx.doi.org/10.1590/S0103-84782007000400016.

BLACKWOOD, L. Cats with cancer: where to start. Journal of Feline Medicine and Surgery, v. 15, n. 5, p. 366-377, 2013. doi: http://dx.doi.org/ 10.1177/1098612X13483235.

BONNETT, B. N.; EGENVALL, A.; HEDHAMMAR, A.; OLSON, P. Mortality in over 350,000 insured Swedish dogs from 1995-2000: I. Breed-, gender-, age- and cause-specific rates. Acta Veterinaria Scandinavica, v. 46, n. 105, p. 105120, 2005. doi: http://dx.doi.org/ 10.1186/1751-0147-46-105.

CRAIG, L. E. Cause of death in dogs according to breed: a necropsy survey of five breeds. Journal of the American Animal Hospital Association, v. 37, n. 5, p. 438-443, 2001. doi: http://dx.doi.org/10.5326/15473317-37-5-438.

EGENVALL, A.; NØDTVEDT, A.; HÄGGSTRÖM, J.; STRÖM HOLST, B.; MÖLLER, L.; BONNETT, B. N. Mortality of Life-Insured Swedish cats during 1999-2006: age, breed, sex, and diagnosis. Journal of Veterinary Internal Medicine, v. 23, n. 6, p. 1175-1183, 2009. doi: http://dx.doi.org/10.1111/j.1939-1676.2009.0396.x.

FIGHERA, R. A.; SOUZA, T. M.; SILVA, M. C.; BRUM, J. S.; GRAÇA, D. L.; KOMMERS, G. D.; IRIGOYEN, L. F.; BARROS, C. S. L. Causas de morte e razões para eutanásia de cães da Mesorregião do Centro Ocidental RioGrandense (1965-2004). Pesquisa Veterinária Brasileira, v. 28 , n. 4 , p. 223-230, 2008. http://dx.doi.org/10.1590/S0100736 X2008000400005.

FLEMING, J. M.; CREEVY, K. E.; PROMISLOW, D. E. L. Mortality in North American dogs from 1984 to 2004: an investigation into age, size and breed-related causes of death. Journal of Veterinary Internal Medicine, v. 25, n. 2, p. 187-198, 2011. doi: http://dx.doi.org/10.1111/j.19391676.2011.0695.x.

FRANCEY, T.; SCHWEIGHAUSER, A. Clinical epidemiology of kidney diseases in the cat. Veterinary Focus, v. 18, n. 2, p. 2-7, 2008.

GONÇALVES, G. A. M; SAlGADO, B. S. Necropsia cosmética em aves. Archives of Veterinary Science, v. 16, n. 2, p. 9-17, 2011. http://dx.doi.org/10.5380/avs.v16i2.17676.
GRAUER, G. F. Urinary tract disorders. In: NELSON, R. W.; COUTO, C. G. (Ed.). Small animal internal medicine. 4. ed. St. Louis, Mo.: Mosby, 2009. p. 607-636; 653-659.

GREENE, G. E.; SCHULTZ, R. D. Immunoprophylaxis. In: GREENE, G. E. (Ed.). Infectious diseases of the dog and cat. St Louis: Saunders, 2006. p. 1069-1118.

HULL, M. J.; NAZARIAN, R. M.; WHEELER, A. E.; BLACK-SCHAFFER, W. S.; MARK, E. J. Resident physician opinions on autopsy importance and procurement. Human Pathology, v. 38, n. 2, p. 342-350, 2007. http://dx.doi.org/10.1016/j.humpath.2006.08.011.

LOSS, L. D.; MUSSI, J. M. S.; MELLO, I. N. K. de; LEÃO, M. S.; FRANQUE, M. P. Responsible pet ownership and dog owners conduct in the county of Alegre-ES. Acta Veterinaria Brasilica, v. 6, n. 2, p. 105-111, 2012.

MARTINS, D. B.; OLIVEIRA, D. T. de; CAMERA, L. Comportamento tumoral em cães. In: SEMINÁRIO INTERINSTITUCIONAL DE ENSINO, PESQUISA E EXTENSÃO, 16., 2011, Cruz Alta. Anais... Cruz Alta, RS: UNICRUZ, 2011.

MENEZES, R. S.; CAMPOS, S. N.; FERREIRA, A. M. R.; ALMEIDA, E. C. P. Retrospective study of causes of death in dogs in private laboratory - RJ. In: ENCONTRO NACIONAL DE PATOLOGIA VETERINÁRIA E I CONGRESSO BRASILEIRO DE PATOLOGIA VETERINÁRIA, 15., 2011, Goiânia. Anais... Goiânia: Patologia Veterinária/EVZ/UFG, 2011.

MOORE, G. E.; BURKMAN, K. D.; CARTER, M. N.; PETERSON, M. R. Causes of death or reasons for euthanasia in military working dogs: 927 cases (1993-1996). Journal of the American Veterinary Medical Association, v. 219, n. 2, p. 209-214, 2001. doi: http://dx.doi.org/10.2460/javma.2001.219.209.

NORTH, S. M.; BANKS, T. A. Small animal oncology: an introduction. Edinburgh: Saunders, 2009. 304 p.

PROSCHOWSKY, H. F.; RUGBJERG, H.; ERSBOLL, A. K. Mortality of purebreed and mixed-breed dogs in Denmark. Preventive Veterinary Medicine, v. 58, n. 1-2, p. 63-74, 2003. doi: http://dx.doi.org/10.1016/S0167-5877(03)00010-2.

SCHERK, M. Urinary tract disorders. In: LITTLE, S. E. (Ed.). The cat: clinical medicine and management. St. Louis, Mo: Saunders, 2012. p. 962-976. 
TRAPP, S.; IACUZIO, A.; BARCA JUNIOR, F.; KEMPER, B.; SILVA, L.; OKANO, W.; TANAKA, N.; GRECCO, F.; CUNHA FILHO, L.; STERZA, F. Causes of death and reasons for euthanasia in a hospital population of dogs and cats. Brazilian Journal of Veterinary Research and Animal Science, v. 47, p. 395-402. 2010. doi: http://dx.doi.org/10.1590/S1413-95962010000500009.
YOUNG, K. Common causes of death in cats. In: WORLD SMALL ANIMAL VETERINARY ASSOCIATION WORLD CONGRESS, 36., 2011, Korea. Proceedings... Jeju, Korea: WSAVA, 2011.

WITHROW, S. Why worry about cancer in pets? In: WITHROW, S. J.; MACEWEN, E. G. (Ed.). Small animal clinical oncology. Philadelphia: Saunders, 2007. p. 15-16. 